Polymer Journal, Vol. 38, No. 7, pp. 659-668 (2006)

(C) 2006 The Society of Polymer Science, Japan

\title{
A Comparative Study of Conductive Polypyrrole and Polyaniline Coatings on Electro-Active Papers
}

\author{
Jaehwan Kim,,${ }^{1, \dagger}$ S. D. DeShPANDE, ${ }^{1,2}$ Sungryul Yun, ${ }^{1}$ and Qubo $\mathrm{LI}^{1}$ \\ ${ }^{1}$ Creative Research Center for EAPap Actuator, Department of Mechanical Engineering, \\ Inha University, Incheon 402-751, South Korea \\ ${ }^{2}$ National Chemical Laboratory, Chemical Engineering Division, Pune-41108, India
}

(Received December 5, 2005; Accepted February 25, 2006; Published May 29, 2006)

\begin{abstract}
A comparative study is performed on the conductive polymer-coated electro-active paper (EAPap) actuators by aiming at improving the overall performance of the actuators. Conductive Polymer coated Electro-active Paper [CP-EAPap] actuators are constructed using electro-deposition of polypyrrole and polyaniline on to cellulose paper. The actuation behavior is tested at different actuation frequency and humidity conditions on bilayer and trilayer types of CP-EAPap actuators. The effect of thickness variation, dopant ions and humidity conditions are compared for both conductive polymer-coated actuators. The trilayer actuators are superior than the bilayer counterparts, and polyaniline coated actuators gives better performance than polypyrrole based ones in terms of bending displacement and ruggedness to humidity. The possible actuation principle is also addressed along with X-ray diffraction and scanning electron microscope results. [doi:10.1295/polymj.PJ2005185]

KEY WORDS Electro-Active Paper / Electro-generation / Polypyrrole / Polyaniline / Bending Actuator /
\end{abstract}

In last ten years, the field of Electro-active polymers (EAP), has received much attention due to the development of new materials that exhibits a large displacement outputs. This characteristic is a valuable attribute that has enabled a myriad of potential applications, and it has evolved to offer operational similarity to biological muscles. Hence, recent studies on EAP research, tends to focus mainly on the development and understanding of the new polymer materials. Some of the presently available materials are ionic polymer metal composites (IPMC), gel polymers, conductive polymers (CP), electrostrictive polymer artificial muscle (EPAM) and so on. ${ }^{1-6}$ Among these conductive polymers has been studied extensively, ${ }^{7-12}$ since they are both ionic and electronic conductive materials that allow the construction of actuators with a basic mechanism similar to natural muscles.

The use of paper as an electrostrictive EAP actuator has been demonstrated by kim et al. ${ }^{1,13}$ Since paper is a sheet that is composed of a fibrous nature, which form a network structure, there is a possibility to prepare a paper that can meet the requirements for EAP actuators. Such actuators were prepared by depositing thin electrodes on both sides of cellulose paper. When an electric voltage was applied to the electrodes the actuator produced a bending displacement. This was termd as Electro-Active Paper (EAPap). EAPap material has advantages in terms of light-weight, dryness, large displacement, low activation voltage, low power consumption and biodegradability. However, the per- formance of EAPap is marginally fit into the range of natural muscles. ${ }^{1}$ Especially, the force output and frequency band improvement of the material are essential.

There are some approaches that can improve the force and frequency band of EAPap material. When the thickness of EAPap material is increased, the stiffness is increased and the force and frequency band can be improved. However, this may require the sacrifice of large displacement output and the low actuation voltage. Conductive polypyrrole polymer has been coated on EAPap material and the tip displacement of the bending actuator has been increased, ${ }^{14,15}$ shown the potential of EAPap material as biomimetic actuators. We termed these devices as conductive polymerelectro-active paper (CP-EAPap) actuators.

Aiming at to improve the performance of CPEAPap actuator, this paper presents of the comparative study on the performance of CP-EAPap actuators fabricated with polypyrrole (PPy) and polyaniline (PANI) conductive polymers. Two types of actuators were constructed viz. $\mathrm{CP} /$ cellulose paper bilayers and $\mathrm{CP} /$ cellulose paper/CP trilayers. Configurations of these devices are shown in Figure 1. The present work describes the synthesis, characterization and actuation performance within these types of actuators. The effect of dopant ion (used in electro-generation of conductive polymer films), film thickness, humidity changes, on actuation behavior has been described. The possible actuation principle is also addressed.

${ }^{\dagger}$ To whom correspondence should be addressed (Tel: +82-32-860-7326, Fax: +82-32-868-1716, E-mail: jaehwan@inha.ac.kr). 


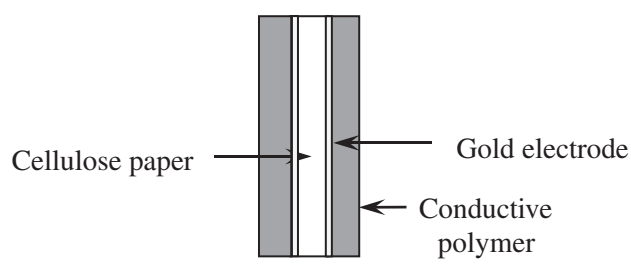

$\underline{\text { Trilayer Actuator Model }}$

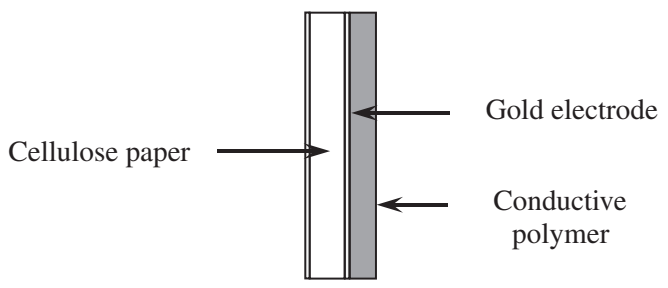

$\underline{\text { Bilayer Actuator Model }}$

Figure 1. Bilayer and trilayer models of conductive polymer coated EAPap.

\section{EXPERIMENTAL}

\section{Materials}

Aniline and Pyrrole (A.R. Aldrich) were used after vacuum distillation. Lithium per chlorate, Lithium tetrafluoro borate, Lithium chloride, Tetrabutyl ammonium hexafluorophosphate $\left(\mathrm{TBAPF}_{6}\right)$, Dichloro Acetic acid (DCA), Propylene Carbonate (PC) Acetonitrile $(\mathrm{ACN})$ were all A.R. grade reagents from Aldrich and were used as received. A commercial cellophane paper available in Korea was used as the cellulose paper in CP-EAPap. The thickness of the paper is $20 \mu \mathrm{m}$. Electrodes are made on the paper with gold by physical vapor deposition. The size of the electrodes is $40 \mathrm{~mm} \times 10 \mathrm{~mm}$ and they are deposited on both sides of the cellulose paper.

\section{Electro-generation of Conductive Polymer Films}

The conductive polyaniline and polypyrrole films were prepared potentiostatically using a Solartron Electrochemical Interface unit [Model SI 1287] at $0.9 \mathrm{~V}$. and $0.7 \mathrm{~V}$. vs. SCE for varying time intervals between $15 \mathrm{~min}$ to $120 \mathrm{~min}$. Figure 2 shows the apparatus setup. All the electro-generation experiments were carried out at room temperature in an inert nitrogen atmosphere. In the conventional three electrodes system, the calomel was used as a reference electrode. The working electrode on which, monomers were polymerized, was composed of a cellulose paper ( $20 \mu \mathrm{m}$ thickness) with gold deposition on both sides of the paper using physical vapor depositor. The thickness of gold layer was $100-150 \mathrm{~nm}$. The thickness of gold layer was estimated from the amount of gold shots in the physical vapor depositor, in which we accumulated the experimental data of gold thickness with the weight of gold shot. The counter electrode was platinum plate. The active area where conductive polymer films obtained was $30 \mathrm{~mm} \times 10 \mathrm{~mm}$. The thickness of the various films obtained was in the range of 5-60 $\mu \mathrm{m}$. The films were rinsed in pure solvent such as propylene carbonate or acetonitrile several times after room temperature and finally dried in vacuum chamber for $4 \mathrm{~h}$. The acid used in the electro-generation of polyaniline was dichloro acetic acid. This was essential to maintain acidity of the electrolyte. Thus, using these electro-generated conductive polymer films on cellulose paper we constructed the bilayer and trilayer actuator devices.

Details of actuator construction and electrical connection are described here. The electrolyte solution from which the polypyrrole films were electro-generated contains $0.2 \mathrm{M}$ pyrrole monomer and $0.1 \mathrm{M}$ $\mathrm{LiClO}_{4}$ in acetonitrile. The use of an organic solvent was necessary since the cellulose paper gets shrunk

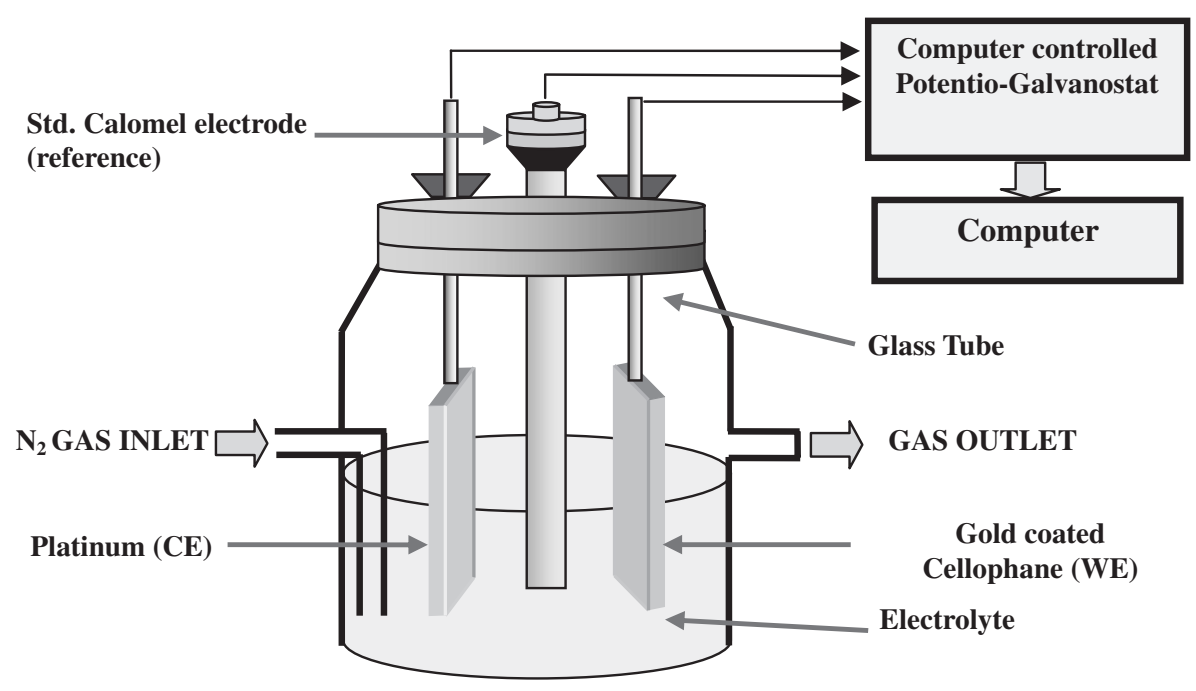

Figure 2. Apparatus of electro-generation process. 
in aqueous medium. Thus, the use of aqueous electrolyte is restricted for the polypyrrole deposition on cellulose paper in the present investigations. In case of polypyrrole films electro-generated using lithium tetrafluoroborate $\left(\mathrm{LiBF}_{4}\right)$ and tetraethyl ammonium hexafluoro phosphate $\left(\mathrm{TEAPF}_{6}\right)$ electrolyte, the same molar ratio to pyrrole monomer, i.e., 2:1 was maintained as described above.

We have used four different dopant ions for the synthesis of polyaniline on to cellulose namely lithium tetrafluoroborate, lithium per chlorate, lithium chloride and tetraethyl ammonium hexafluoro phosphate. We could successfully get only $\mathrm{ClO}_{4}{ }^{-}$and $\mathrm{BF}_{4}{ }^{-}$doped polyaniline films in the presence of DCA in PC. The attempt to get polyaniline films using tetraethyl ammonium hexafluoro phosphate and lithium chloride was unsuccessful. This can be explained by the fact that the lithium chloride was sparingly soluble in PC. There seems to be no dissociation of this salt in PC, hence, it prevented to form any conductive polyaniline at the electrode surface.

\section{Performance Test}

The linear displacement measurement of various film samples was carried out using Laser Doppler Vibrometer (LDV) described in detail elsewhere. ${ }^{15}$ Compurized displacement measurement system was established. This system consists of high precision LDV (Brüe \& Kjær, 8336), an environmental chamber (KMS, CTH3-2S) and function generator (Agilent, 33220A). The LDV catches the tip velocity of the actuator and the analog velocity signal is converted into a digital signal through an A/D board. LabVIEW software in the computer converts the tip velocity into displacement by integrating it over time. Sample actuators were placed in the environmental chamber that can control the humidity (30-95\% RH, relative humidity) and temperature $\left(10-100^{\circ} \mathrm{C}\right)$. Samples were held in a gripper that has electric contacts wired to the function generator and placed in the chamber in vertical direction. The electrical voltage generated from the function generator was fed to the gold electrodes of CP-EAPap actuators. The actuators were tested for its performance in air at different humidity and actuation frequencies conditions with room temperature $\left(23^{\circ} \mathrm{C}\right)$. The displacement is recorded in terms of the amplitude of bending displacement at the tip of the actuators.

\section{RESULTS AND DISCUSSION}

The PPy and PANI coated EAPap actuators were made by the electro-generation with different dopants. During the PANI polymerization process, the films for $\mathrm{PF}_{6}{ }^{-}$and $\mathrm{Cl}^{-}$doped form couldn't get into practice due to its nature. The corresponding salt did not get dissolved into solution, and hence it was not polymerized into desired format. The adhesion of conductive polymers to a metal substrate is of prime importance in many applications where good electrical contact and durability of the system is required. The adhesion of CP-EAPap was examined by the peel-off test performed on freshly prepared (4h after electrochemical deposition and subsequent drying films) and aged films 4 months after the synthesis. The adhesion between gold and conductive polymer was very good where as cellulose-gold adhesion was comparatively poor according to the peel-off test of scotch tape. The cellulose-gold poor adhesion was obvious due to the vapor deposition method used for depositing thin gold layer $(100-150 \mathrm{~nm})$ on to cellulose paper.

\section{Characterization of CP-EAPap Actuator}

The conductive polymer films deposited on cellulose paper for different time intervals gave different film thickness. In the case of polyaniline, the thickness was linearly varied from 5 to $60 \mu \mathrm{m}$ with increase in deposition time from 15 to 60 min with different dopant ions. The $\mathrm{ClO}_{4}{ }^{-}$doped films are thicker (15 to $60 \mu \mathrm{m})$ than $\mathrm{BF}_{4}{ }^{-}$doped films $(5-40 \mu \mathrm{m})$. In the case polypyrrole, the deposition time was from 30 to $120 \mathrm{~min}$, and the thickness was almost linearly increased with the time. Since the thickness of PPy deposition was small, the deposition time was increased twice. The film thickness obtained using $\mathrm{ClO}_{4}{ }^{-}$ and $\mathrm{BF}_{4}{ }^{-}$dopant ions were 7 to $23 \mu \mathrm{m}$ thick where as $\mathrm{PF}_{6}{ }^{-}$doped films were thicker, about $20-70 \mu \mathrm{m}$. In the next section, the effect of film thickness on actuation performance is discussed in detail. Figure 3 shows the cross-section SEM micrograph of PPy$\mathrm{BF}_{4}{ }^{-}$-EAPap bilayer actuator. The thickness of the conductive polymer layer was about $30 \mu \mathrm{m}$. The micrograph shows the film formation of the conductive polymer with the cellulose paper.

Figure 4 represents SEM pictures taken from the surface of the PANI and PPy samples doped with $\mathrm{BF}_{4}{ }^{-}$ions. Both of them show a compact morphology. The electro-synthesized PANI in propylene carbonate medium in the presence of dichloro-acetic acid exhibited a compact structure, similar to that obtained by Yonezawa et al. ${ }^{16}$ Thus, the compact morphology in PC medium is quite expected, as this kind of bonding effect is absent in the case of PC, which is a typical example of a non-associated solvent. On the other hand, the polyaniline doped with $\mathrm{ClO}_{4}{ }^{-}$ showed some porosity when compared with the $\mathrm{BF}_{4}{ }^{-}$ doped one. The PANI synthesized in aqueous medium in the presence of DCA and $\mathrm{LiClO}_{4}$ has been reported to have globular morphology due to hydrogen bonding effect. ${ }^{17}$ 


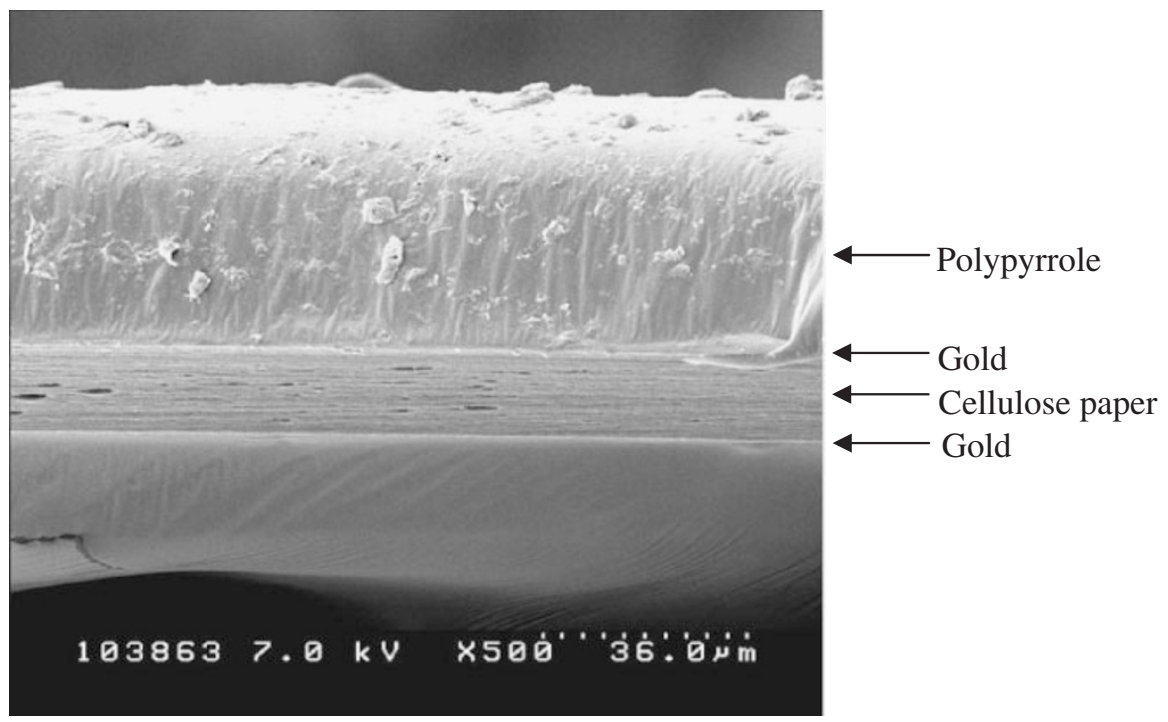

Figure 3. SEM micrograph of CP-EAPap actuator (cross section) constucted with $\mathrm{PPy}_{-\mathrm{BF}_{4}}{ }^{-}$doped $90 \mathrm{~min}$ film.

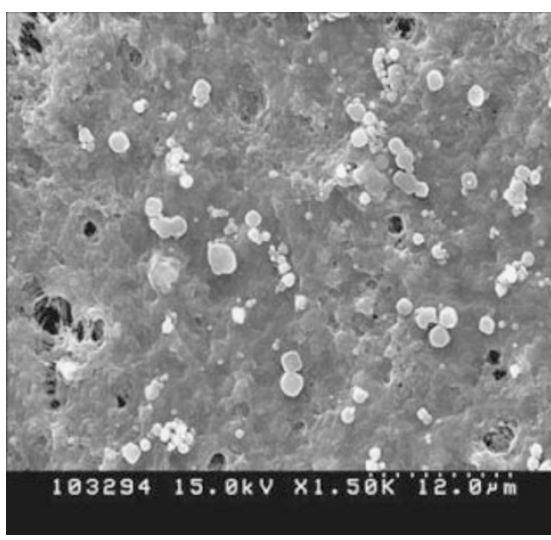

(A)

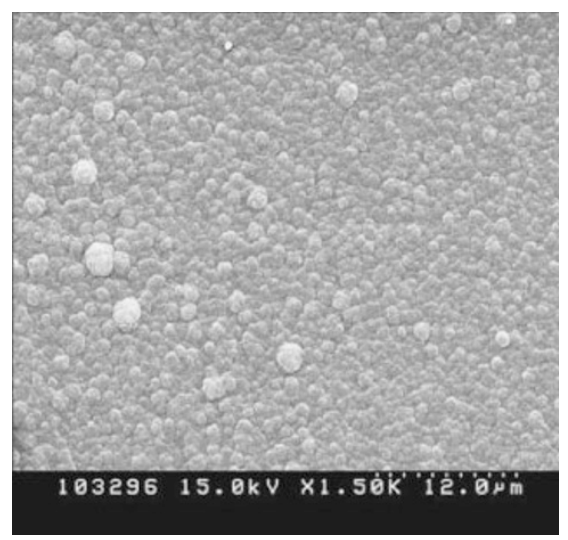

(B)

Figure 4. SEM micrographs of $\mathrm{A}$ : PANI film $\mathrm{BF}_{4}{ }^{-}$doped and $\mathrm{B}$ : $\mathrm{PPy}$ film $\mathrm{BF}_{4}{ }^{-}$doped.

\section{Actuation Performance in Air}

The actuation behavior of bilayer and trilayer devices was investigated in air medium. All these measurements were carried out in the environment chamber. The test voltage from a function generator was chosen to be $6 \mathrm{~V}_{\mathrm{p}-\mathrm{p}}$ (peak to peak) with frequency changes from $0.5 \mathrm{~Hz}$ to $7 \mathrm{~Hz}$. It was observed that at $90 \%$ humidity maximum displacement was obtained in each of the tested samples.

Figures 5 and 6 show bending displacement amplitudes of PPy-EAPap actuators with different dopants $\left(\mathrm{ClO}_{4}{ }^{-}\right.$and $\left.\mathrm{BF}_{4}{ }^{-}\right)$and deposition times. Horizontal axis is the actuation frequency.

Table I shows maximum displacements of PPy-EAPap actuators with different configurations, deposition times and dopants. Trilayer configuration of PPy$\mathrm{BF}_{4}{ }^{-}$-EAPap actuator showed the maximum displacement of $10.5 \mathrm{~mm}$ at $90 \mathrm{~min}$ deposition time. In the bilayer configuration, the dopant effect was small, but it was large in the trilayer configuration: the $\mathrm{BF}_{4}{ }^{-}$ dopant case showed the highest displacement $10.5 \mathrm{~mm}$ while the $\mathrm{ClO}_{4}{ }^{-}$dopant case did $5.7 \mathrm{~mm}$. This may be due to the different doping levels obtained from different dopant ions. The smallest dopant of these is $\mathrm{BF}_{4}{ }^{-}$ ion, which shows more conductivity. Therefore, the PPy-EAPap actuators made with $\mathrm{BF}_{4}{ }^{-}$dopant were better than the $\mathrm{ClO}_{4}{ }^{-}$dopant devices. There is an optimum thickness (deposition time) of PPy layer that maximizes the bending displacement. 60 and $90 \mathrm{~min}$ deposition times for bilayer and trilayer configurations showed the maximum displacement, while the highest thickness films (120 min deposited) showed the less displacement. It is since thicker films can cause opposing force to the displacement due to the increased mass of films. Generally, the resonance frequency is linearly increased with the thickness of film. In Figures 5 and 6, the maximum displacement peaks were occurred at resonance frequencies of PPy-EAPap actuators, and these frequencies tend to be increased with the deposition time, albeit some deviations are 
(A)

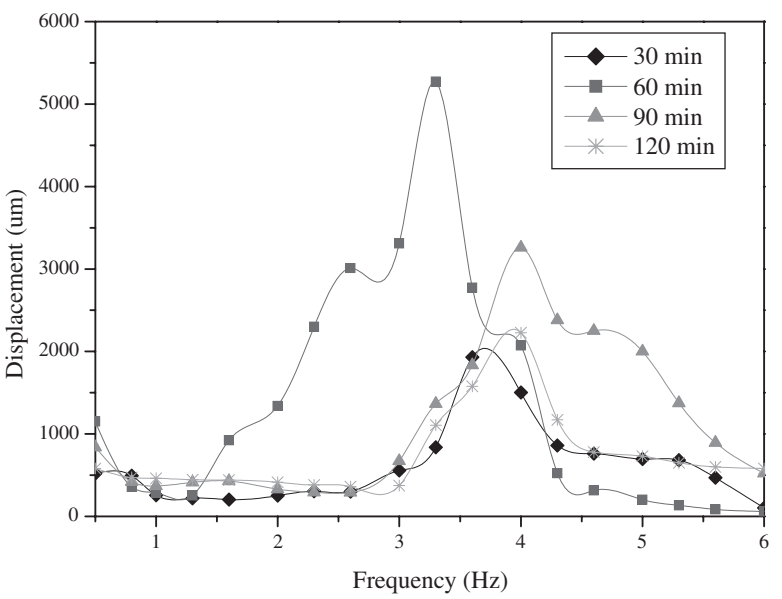

(B)

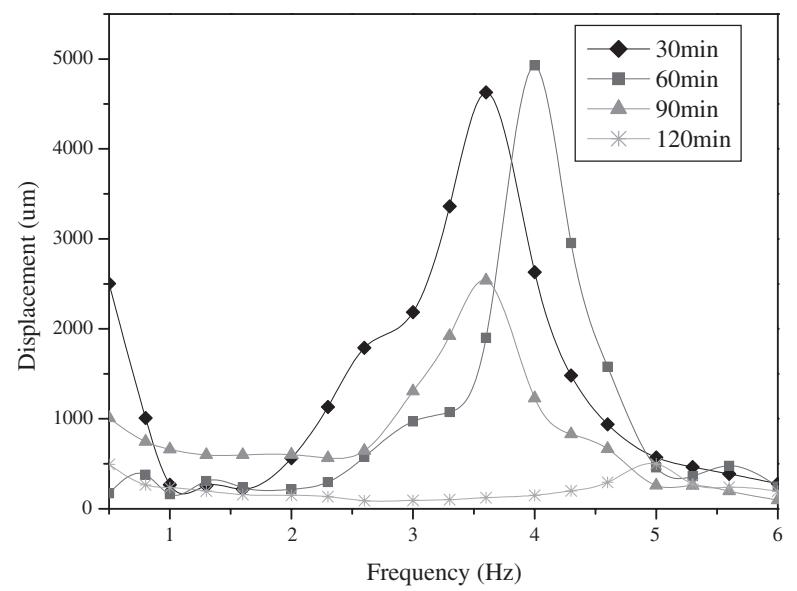

Figure 5. Displacement of PPy coated EAPap bilayer devices with different deposition time. (A) $\mathrm{BF}_{4}{ }^{-}$dopant and (B) $\mathrm{ClO}_{4}{ }^{-}$ dopant.

seen. These deviations may be associated with inhomogeneity of the coated layers with the deposition time.

Figures 7 and 8 show bending displacement amplitudes of PANI-EAPap actuators with different dopants $\left(\mathrm{ClO}_{4}{ }^{-}\right.$and $\left.\mathrm{BF}_{4}{ }^{-}\right)$and deposition times. Table II exhibits maximum displacements of PANI-EAPap actuators. Maximum displacements of most actuators were happened at $45 \mathrm{~min}$ deposition time except the

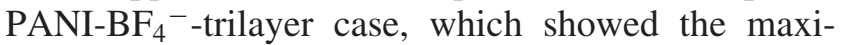
mum displacement of $10.1 \mathrm{~mm}$. The PANI-EAPap actuator made with $\mathrm{ClO}_{4}{ }^{-}$dopant was better than the $\mathrm{BF}_{4}{ }^{-}$doped actuator.

To investigate this difference, SEM pictures were taken on the surfaces of PANI-BF ${ }_{4}^{-}$-EAPap and PANI- $\mathrm{ClO}_{4}{ }^{-}$-EAPap actuators (Figure 9). Bigger

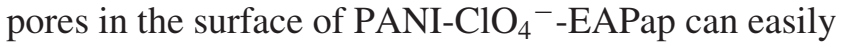
allow ions penetrate through the films more freely. A porous nature of the PANI-EAPap doped with $\mathrm{ClO}_{4}{ }^{-}$ may result in good actuation.

In the bilayer configuration, the maximum displacement of polypyrrole coated bilayer actuator (60 min deposited) was about $5.2 \mathrm{~mm}$, whereas, the poly-
(A)

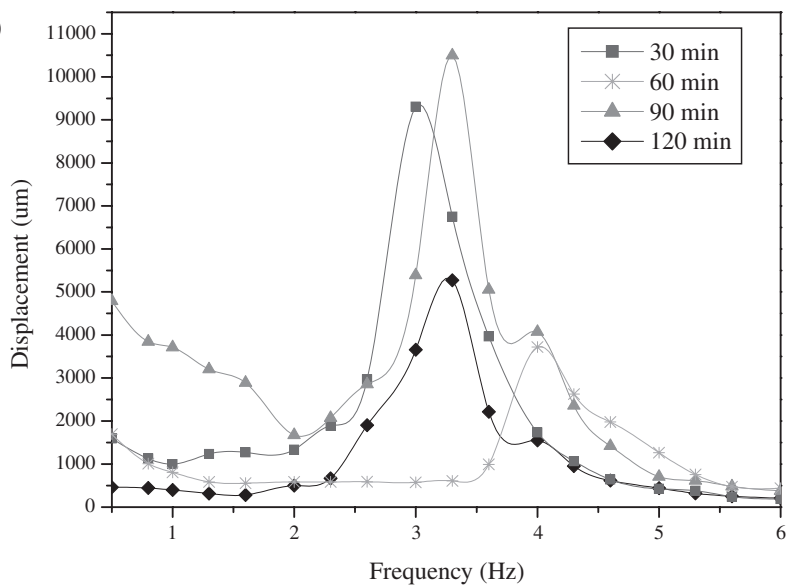

(B)

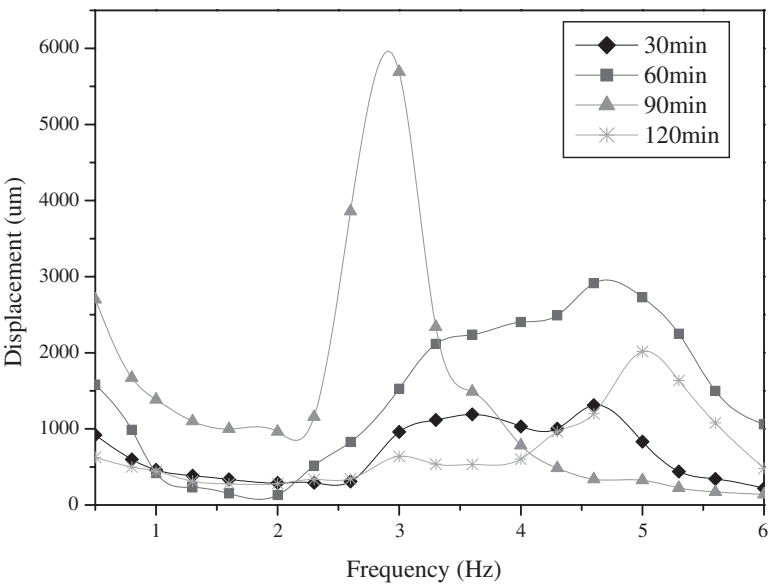

Figure 6. Displacement of PPy coated EAPap trilayer devices with different deposition time. (A) $\mathrm{BF}_{4}{ }^{-}$dopant and (B) $\mathrm{ClO}_{4}{ }^{-}$ dopant.

Table I. Displacement (mm) of PPy coated EAPap actuators (with configurations, deposition times of PPy and dopants)

\begin{tabular}{cccc}
\hline Configuration/Coating Time & $\mathrm{BF}_{4}{ }^{-}$dopant & $\mathrm{ClO}_{4}{ }^{-}$dopant \\
\hline \multirow{4}{*}{ Bilayer } & $30 \mathrm{~min}$ & 1.9 & 4.6 \\
& $60 \mathrm{~min}$ & 5.2 & 4.9 \\
& $90 \mathrm{~min}$ & 3.3 & 2.5 \\
& $120 \mathrm{~min}$ & 2.2 & 1.5 \\
\hline \multirow{6}{*}{ Trilayer } & $30 \mathrm{~min}$ & 5.8 & 1.3 \\
& $60 \mathrm{~min}$ & 9.3 & 2.9 \\
& $90 \mathrm{~min}$ & 10.5 & 5.7 \\
\hline
\end{tabular}

aniline coated counterpart ( $45 \mathrm{~min}$ deposited) showed this value as $6.7 \mathrm{~mm}$. The experimental condition for tests was identical for both samples. As the polypyrrole films were thinner than the polyaniline counterparts, the deposition time of polypyrrole films was taken longer. The PANI- $\mathrm{ClO}_{4}{ }^{-}$-EAPap actuator was the best one in the bilayer configuration. This is mainly due to the electronic configuration of polyaniline, which is more stable than PPy.

In the trilayer configuration, it was found out that the trilayer devices were more in actuation as com- 
(A)

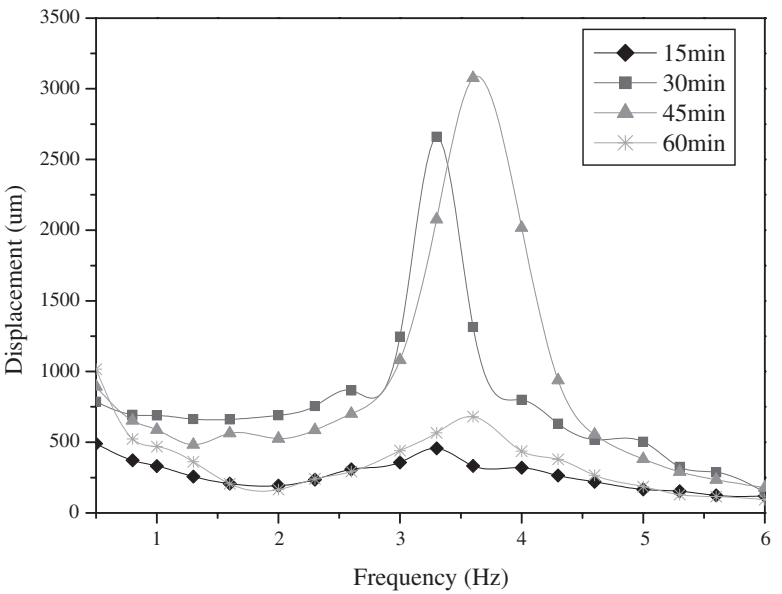

(B)

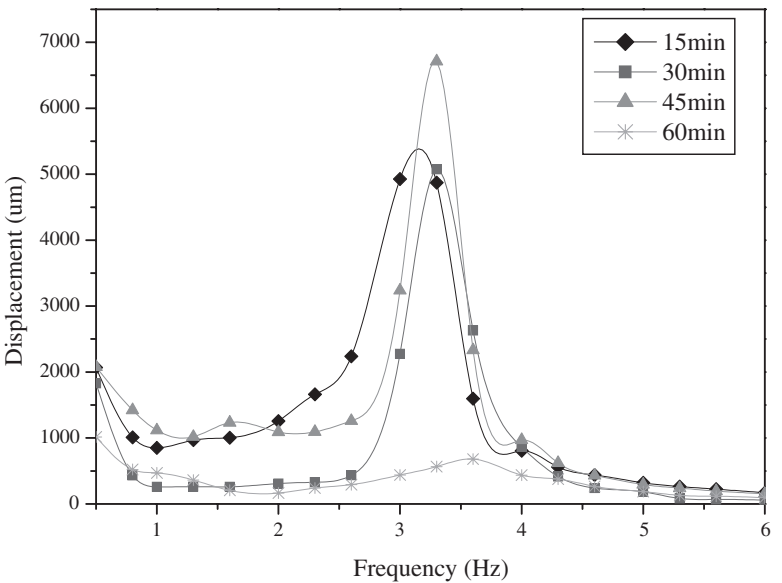

Figure 7. Displacement of PANI coated EAPap bilayer devices with different deposition time. (A) $\mathrm{BF}_{4}{ }^{-}$dopant and (B) $\mathrm{ClO}_{4}{ }^{-}$dopant.

pared to bilayer devices. The trilayer devices have advantage of symmetry on both sides of EAPap. Both sides will actuate the device more accurately as compared to other bilayer devices, resulting in the trilayer devices to get more actuation. In the trilayer configuration, the $\mathrm{PPy}_{-} \mathrm{BF}_{4}{ }^{-}$-EAPap actuator showed the maximum displacement $10.5 \mathrm{~mm}$, while the PPy$\mathrm{ClO}_{4}{ }^{-}$-EAPap actuator did $5.7 \mathrm{~mm}$. Meanwhile, PANI-EAPap counterparts exhibited more than 10.0 $\mathrm{mm}$ of the maximum displacement. It is known that polyaniline films are more stable than PPy counterparts. Also, we have known that the crystalline peak area obtained during the synthesis of the devices was converted into amorphous region as can be seen from the after actuation. This will be discussed in the next section. In overall, the displacement output of PANIEAPap actuators was better than the PPy-EAPap actuators.

The humidity effect on CP-EAPap actuators was investigated. Figure 10 shows the displacement of the $\mathrm{PPy}-\mathrm{BF}_{4}$-trilayer case (90 minutes deposited) at $70 \%, 80 \%$ and $90 \%$ relative humidity (RH) levels. As the humidity level was increased, the displacement
(A)

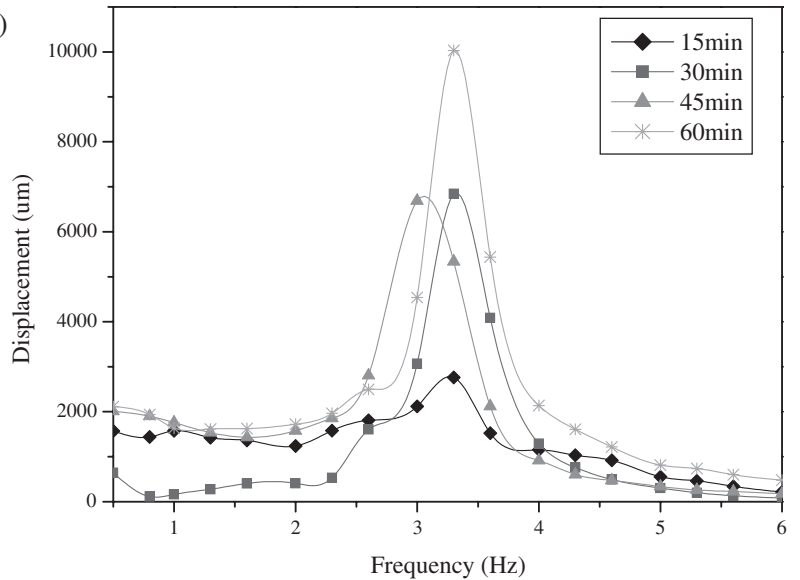

(B)

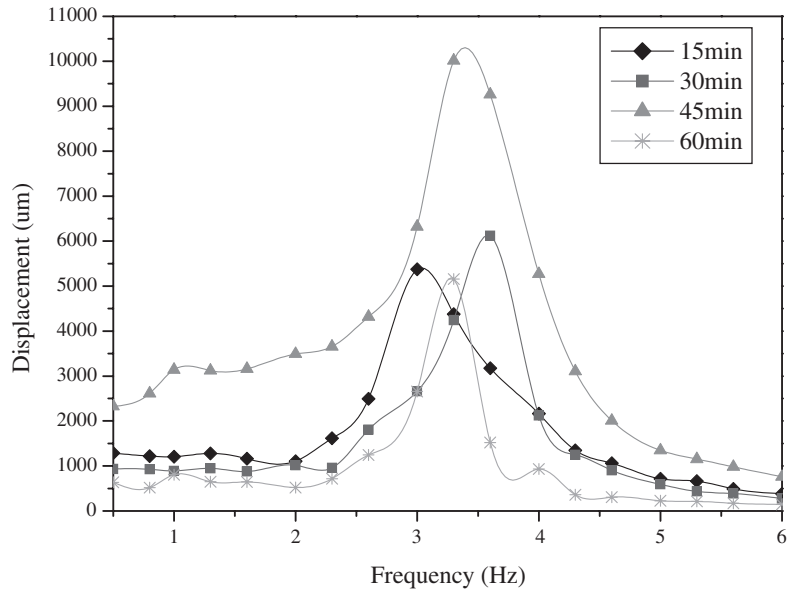

Figure 8. Displacement of PANI coated EAPap trilayer devices with different deposition time. (A) $\mathrm{BF}_{4}^{-}$dopant and (B) $\mathrm{ClO}_{4}^{-}$dopant.

Table II. Displacement (mm) of PANI coated EAPap actuators (with configurations, deposition times of PANI and dopants)

\begin{tabular}{cccc}
\hline Configuration/Coating Time & $\mathrm{BF}_{4}{ }^{-}$dopant & $\mathrm{ClO}_{4}{ }^{-}$dopant \\
\hline \multirow{4}{*}{ Bilayer } & $15 \mathrm{~min}$ & 0.5 & 4.9 \\
& $30 \mathrm{~min}$ & 2.7 & 5.1 \\
& $45 \mathrm{~min}$ & 3.1 & 6.7 \\
& $60 \mathrm{~min}$ & 0.7 & 3.8 \\
\hline \multirow{4}{*}{ Trilayer } & $15 \mathrm{~min}$ & 2.8 & 4.4 \\
& $30 \mathrm{~min}$ & 6.9 & 6.9 \\
& $45 \mathrm{~min}$ & 6.7 & 10.0 \\
& $60 \mathrm{~min}$ & 10.1 & 5.2 \\
\hline
\end{tabular}

was increased, and the maximum displacement $10.5 \mathrm{~mm}$ was observed. Notice that the resonance frequency where the peak displacement located, was decreased as the humidity level was increased. This was due to the fact that the increased humidity added more mass on the device so as to reduce the resonance frequency. Also, the stiffness of cellulose paper was decreased as the humidity level was increased, which resulted in the resonance frequency decrease. Different humidity data obtained from PANI-BF 4 -trilayer case is shown in Figure 11. It can be shown that at $60 \%$ 


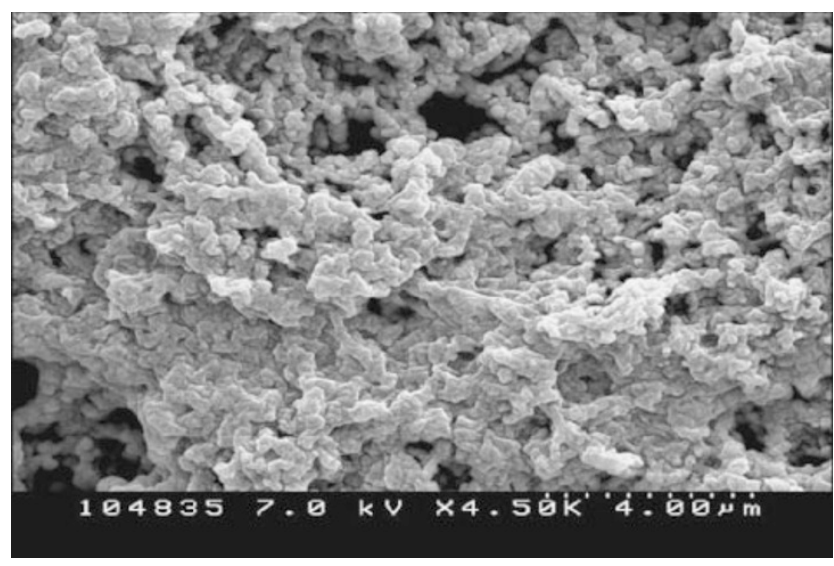

(A)

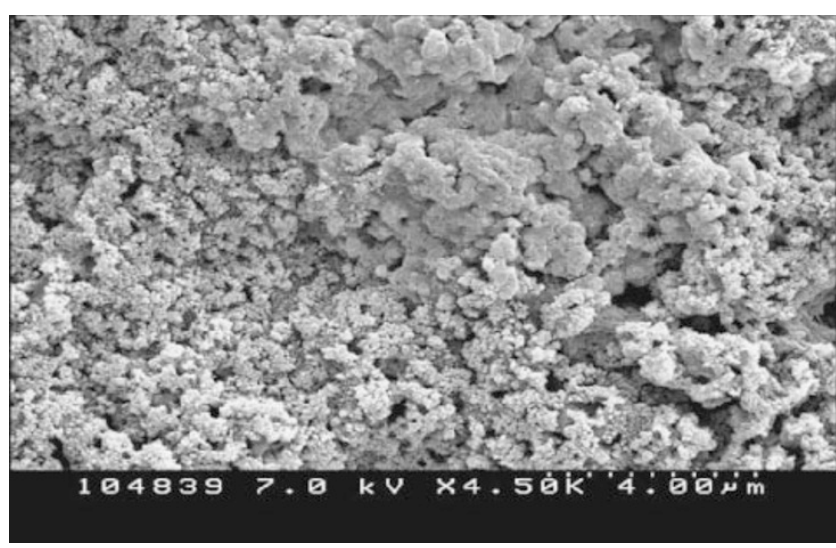

(B)

Figure 9. SEM pictures of PANI samples: A: $\mathrm{ClO}_{4}{ }^{-}$doped and $\mathrm{B}: \mathrm{BF}_{4}{ }^{-}$doped.

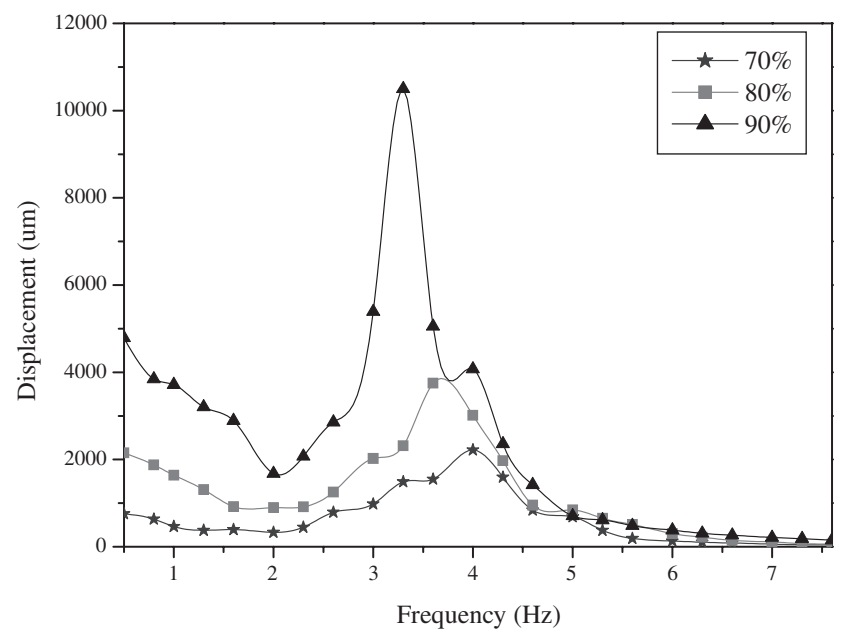

Figure 10. The effect of relative humidity on CP-EAPap actuator ( $\mathrm{PPy}, \mathrm{BF}_{4}{ }^{-}$doped trilayer, $\left.90 \mathrm{~min}\right)$.

$\mathrm{RH}$ the displacement was the minimum. The data at $90 \%$ and $95 \%$ humidity was almost similar to getting at saturation. At $90 \% \mathrm{RH}$, the maximum displacement of $10.1 \mathrm{~mm}$ was attained. A remarkable result is that the humidity effect on the PANI-BF 4 -trilayer case

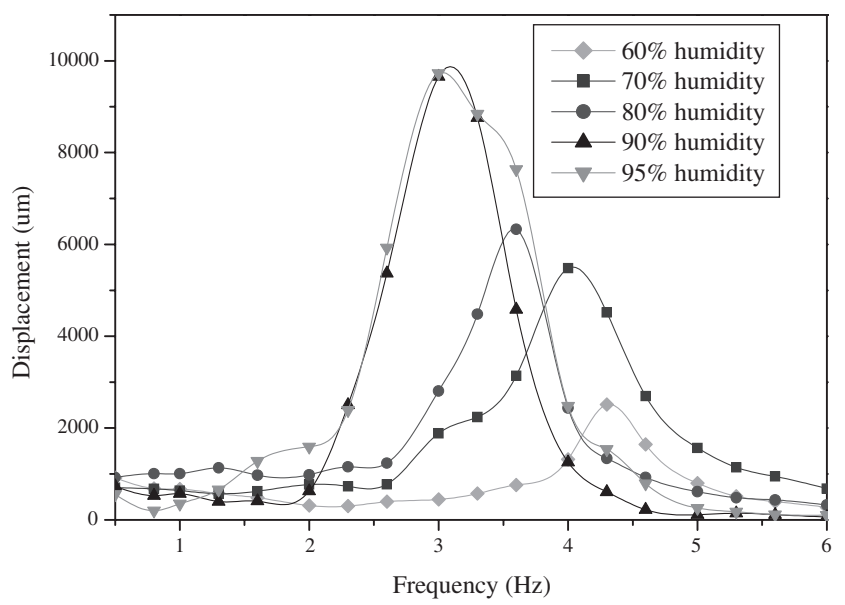

Figure 11. The effect of relative humidity on CP-EAPap actuator (PANI, $\mathrm{BF}_{4}{ }^{-}$doped trilayer, $60 \mathrm{~min}$ ).

is small. For example, if the relative humidity level is above $80 \%$, the PANI- $\mathrm{BF}_{4}{ }^{-}$-EAPap actuator can achieve nearly $70 \%$ of the maximum displacement.

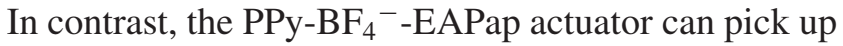
only $20 \%$. Thus, the actuation performance of PANIEAPap actuators is better than that of PPy-EAPap actuators. There are many reasons for this to explain. The first one is the crystalline nature of the polyaniline film, which changes to amorphous nature after the actuation. The second is the high conductivity of polyaniline samples than the polypyrrole samples.

\section{Working Principle}

The base material of cellulose has been reported to possess piezoelectric properties. ${ }^{18}$ Partial orientation of the cellulose fiber in the paper pulp is primarily the reason of piezoelectric properties in papers. On the other hand, the cellulose paper mainly consists of cellulose in its amorphous state and the hydroxyl groups in it can easily interact with polar compounds. Thus, for only EAPap actuator, the combined effect of piezoelectric and ion migration associated with dipole moments may be an important role in the actuation principle. However, when conductive polymer is coated on the cellulose paper, the actuation principle may be different. To investigate the actuation phenomenon of CP-EAPap materials, several tests were performed in terms of XRD measurement before and after actuation, and film characteristics from SEM and microscope pictures.

Figure 12 shows the $\mathrm{X}$-ray diffraction patterns of PANI-EAPap samples doped with $\mathrm{BF}_{4}{ }^{-}$and $\mathrm{ClO}_{4}{ }^{-}$. In each sample, two curves of before and after actuations were compared. The deposition time of the PANI layer was $30 \mathrm{~min}$. In the PANI-EAPap sample doped with $\mathrm{BF}_{4}^{-}$(Figure 12: A), three XRD peaks at $9.6^{\circ}, 19.2^{\circ}$ and $44.5^{\circ}$ were slightly reduced while the peak at $21^{\circ}$ was a little bit increased. The $38.2^{\circ}$ 
(A)

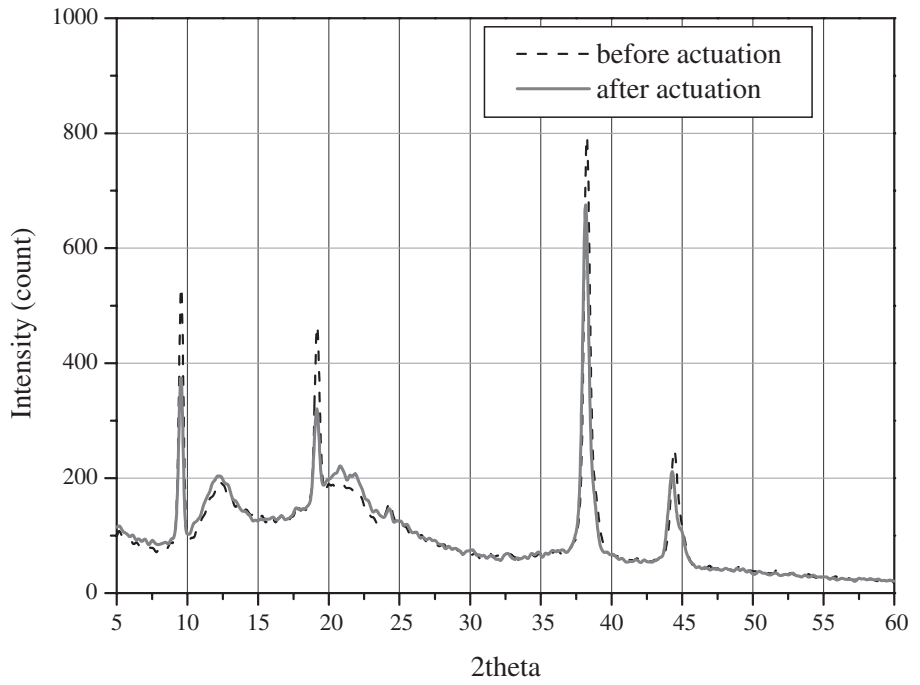

(B)

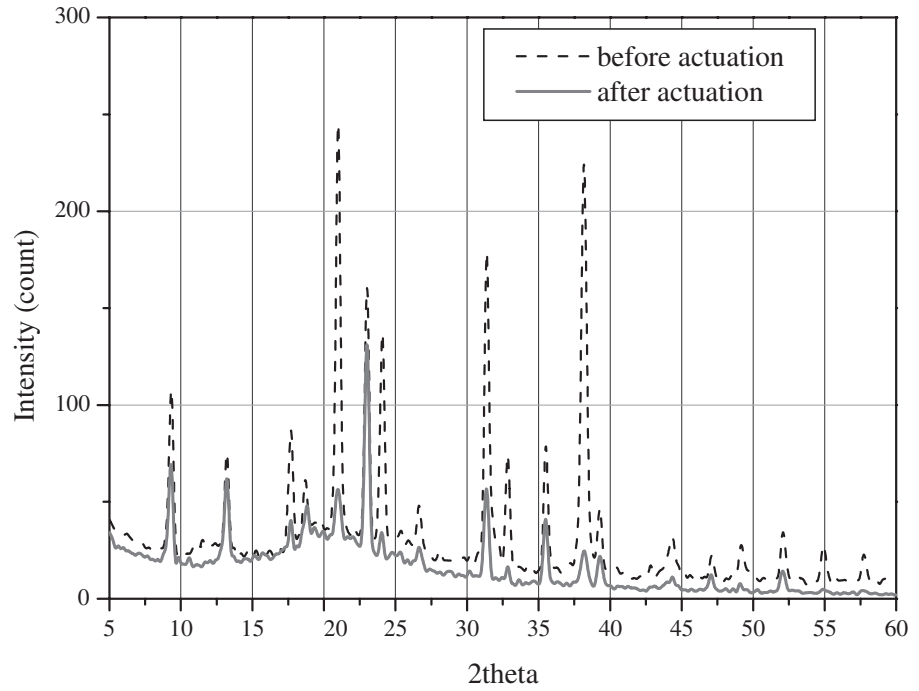

Figure 12. XRD patterns of PANI-EAPap films: A: doped with $\mathrm{BF}_{4}{ }^{-}$ion and $\mathrm{B}$ : with $\mathrm{ClO}_{4}{ }^{-}$ion.

Table III. The XRD peak comparison for PANI coated EAPap $\left(-\mathrm{ClO}_{4}{ }^{-}\right.$dopant $)$

\begin{tabular}{cccccccccccc}
\hline $2 \theta$ & 9.3 & 13.3 & 17.7 & 18.6 & 21.0 & 23.0 & 24.1 & 31.4 & 32.9 & 35.5 & 38.2 \\
\hline $\begin{array}{c}\text { Intensity, } \\
\text { (before actuation) }\end{array}$ & 121 & 91 & 103 & 76 & 278 & 206 & 165 & 217 & 94 & 94 & 276 \\
\hline $\begin{array}{c}\text { Intensity, } \\
\text { (after actuation) }\end{array}$ & 89 & 76 & 88 & 55 & 62 & 166 & 105 & 55 & 57 & 53 & 31 \\
\hline
\end{tabular}

peak was associated with the gold layer coated on the cellulose paper. The $21^{\circ}$ peak may be related with the (200) direction of cellulose crystalline. ${ }^{19}$ This kind of peaks change was more distinct in the $\mathrm{ClO}_{4}{ }^{-}$doped case (Figure 12: B).

Table III shows the comparison of XRD peaks of the PANI- $\mathrm{ClO}_{4}$-EAPap before and after the actuation. There was a decrease in crystalline area within the sample after the activation, which means an increase of amorphous part. Notice that $21^{\circ}$ and $31.4^{\circ}$ peaks were remarkably reduced. The $31.4^{\circ}$ peak was from the PANI doped with $\mathrm{ClO}_{4}{ }^{-}$ion. The suppression of two peaks may be associated with the structural changes taken place in conductive polymer film and cellulose paper due to the application of electrical field. It is quite possible that the crystalline and amorphous segments have been rearranged under the applied electric filed, contributing towards the enhanced the bending displacement. To which content the crystalline part has congregated is unknown, and more investigation on this change is under study.

Further investigation of structural changes was observed using microscopic view of the actuator films before and after the actuation. Figure 13 shows micro- 


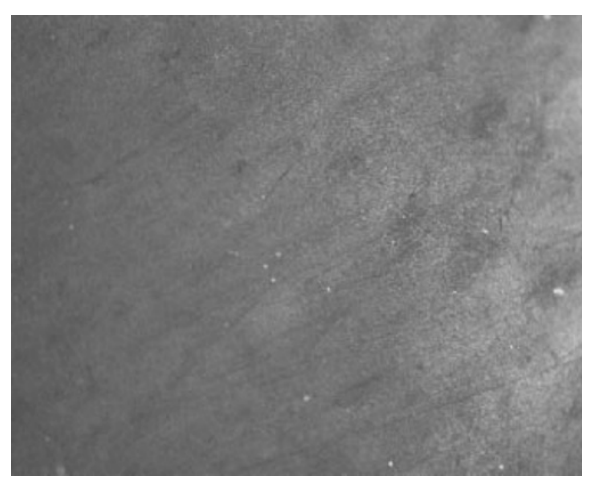

(A)

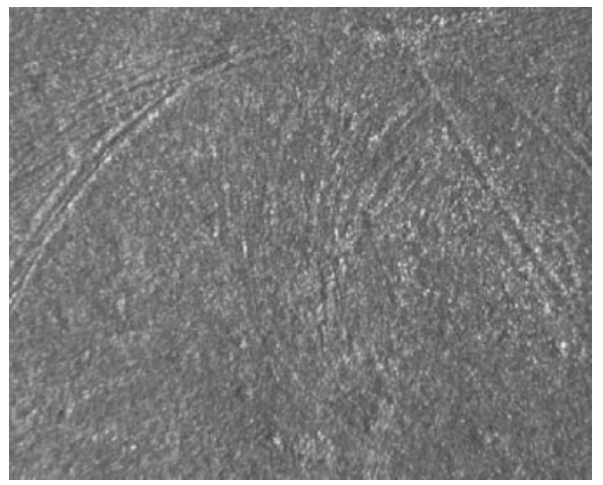

(C)

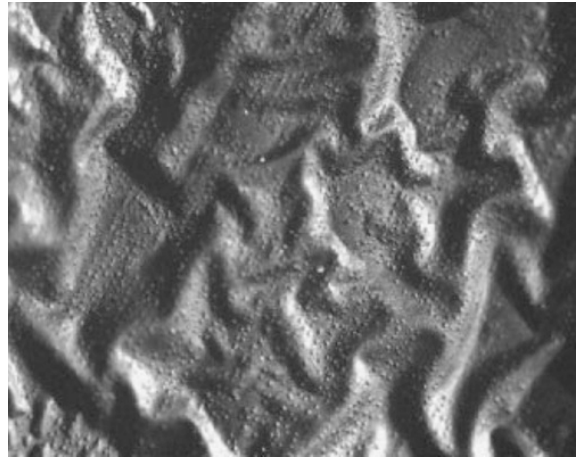

(B)

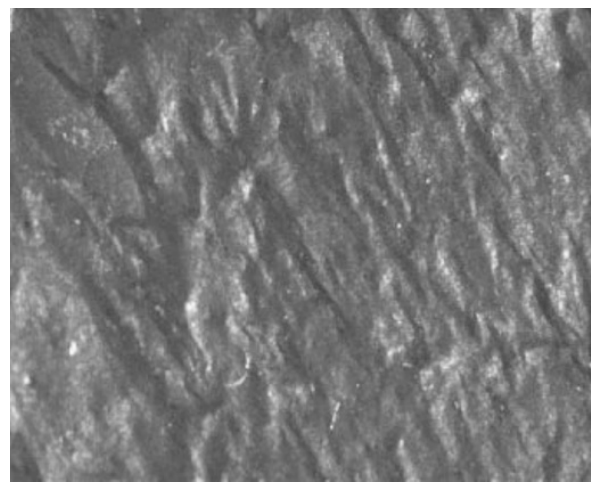

(D)

Figure 13. Microscopic view of CP-EAPap actuator: A: PPy film (Before Actuation, x100) B: PPy film (After actuation, x400), C: PANI film (Before Actuation, x100) and D: PANI film (After Actuation, x400).

scopic views of CP-EAPap actuators: A: PPy film (before actuation), B: PPy film (after actuation), C: PANI film (before actuation) and D: PANI film (after actuation). The actuator samples before activation showed a compact morphology. On the other hand, the same samples after actuation exhibited large bubbles on the surface at a certain local region. The surface also showed many tiny bubble pockets, which were due to movement of gases or moistures in the cellulose paper. The probable source of this bubble formation may be electrolytic gas formation at the metal polymer interface. This observation indicates that a part of the actuation mechanism for CP-EAPap actuators is likely based on a migration effect. Similar type of observation was obtained in our group's earlier investigation on paper actuators. ${ }^{13}$ In the meantime, the motion of coated conductive polymer films lies in a reversible sorption of water vapor, which causes an expansion and contraction of the film. Water molecules in air may penetrate through the conductive polymer layer in the positive electrode during the biased field and expel the molecules in the other electrode to compensate the charge. The corresponding volume may increase for the positive bias and decrease for the negative bias. According to our experimental result, the humidity greatly affects the efficiency of the actuator, and the water molecules may be the dominant factor to operate actuator. Thus, there were elongation and contraction of conductive polymers coated on the surface of EAPap material associated with the water molecules in the air.

The working principle of actuator is mainly divided into two parts. For the EAPap actuator only, the combined effect namely the electrostriction and migration associated with dipole moment of the constituents of cellulose paper plays an important role. However, in the present studies we have used conductive polyaniline and polypyrrole as an active component of the device.

So, in the working principle of CP-EAPap actuators there are additional two factors, which contributed towards the actuation behavior. First factor is the elongation and contraction changes in conductive polymers due to applied currents causing adsorption and desorption of water molecules in the film structure, the second factor, is the structural changes in conductive polymer films due to the application of electrical field. These elongation and contraction result in improved performance of the actuators.

\section{SUMMARY}

Conductive Polymer-Electro-active Paper actuators were constructed using electro deposition of polypyr- 
role and polyaniline on to cellulose paper, and the bending displacement performance of them was compared. The $\mathrm{ClO}_{4}{ }^{-}$and $\mathrm{BF}_{4}{ }^{-}$dopants were used for polypyrrole and polyaniline in non-aqueous solvent medium. The thickness of conductive polymer films deposited on cellulose paper varied almost linearly with the deposition time.

The actuation behavior was tested at different actuation frequency and humidity conditions on bilayer and trilayer configurations of CP-EAPap actuators. The trilayer actuator found superior than the bilayer counterparts. The effect of thickness variation, dopant ion and humidity conditions were compared for both the conductive polymers. As the humidity level was increased, the displacement of CP-EAPap actuators was increased. The polyaniline-coated EAPap actuators gave better performance than the polypyrrolecoated EAPap actuators. This may be due to the crystalline nature of the polyaniline film, which changes to amorphous nature after the actuation, and the relatively high conductivity of polyaniline than the polypyrrole.

The devices were characterized using XRD and SEM techniques before and after the electrical actuation. The suppressed nature of X-ray pattern indicated that the structural changes have taken place in conductive polymer film due to the application of electrical field. This structural change might contribute towards the enhanced tip displacement. The further investigation of structural changes was observed using microscopic view of the actuator films before the actuation and after the actuation. The sample after actuation showed tiny bubble pockets on the surface, indicating that a part of actuation mechanisms for CP-EAPap actuators is likely based on migration effect.

The working principle of CP-EAPap actuators is divided into two parts: i) the combined effect of the electrostriction and migration associated with dipole moment of the constituents of cellulose paper, and ii) the structural changes in conductive polyaniline due to applied currents causing adsorption and desorption of water in the film structure.
Acknowledgment. This work was conducted under the support of Creative Research Initiatives of Korea Science and Engineering Foundation.

\section{REFERENCES}

1. "Electroactive Polymer (EAP) Actuators as Artificial Muscles: Reality, Potential and Challenges," Y. Bar-Cohen, Ed. SPIE press, Washington, 2001.

2. M. Shahinpoor, Y. Bar-Cohen, O. J. Simpson, and J. Smith, Smart Mater. Struct., 7, R-15 (1998).

3. R. Pelrine, R. Kornbluh, and J. Joseph, Sens. Actuators, A, 64, 77 (1998).

4. Q. M. Zhang, V. Bharti, and X. Zhao, Science, 280, 2101 (1998).

5. P. Calvert and Z. Liu, Acta Mater., 46, 2565 (1998).

6. R. Heydt, R. Kornbluh, R. Pelrine, and V. Mason, J. Sound \& Vibration, 215, 297 (1998).

7. R. H. Baughman, L. W. Shacklette, R. L. Elsenbaumer, E. J. Plichta, and C. Becht, in "Conjugated Polymeric Materials: Opportunities in Electronics, Optoelectronics and MoleElectronics," J. L. Bredas and R. R. Chance, Ed., Kluwer, Dordrecht, 1999, p 559.

8. Q. Pei and O. Inganas, Adv. Mater., 4, 277 (1992).

9. E. Smela, O. Inganas, and L. Lundstrom, Science, 268, 1735 (1995).

10. W. Takashima, M. Kaneko, K. Kaneto, and A. G. MacDiarmid, Synth. Met., 71, 2265 (1995).

11. T. F. Otero and J. M. Sansinena, Adv. Mater., 10, 491 (1998).

12. J. D. Madden, R. A. Cush, T. S. Kanigan, and I. W. Hunter, Synth. Met., 113, 185 (2000).

13. J. Kim and Y. B. Seo, Smart Mater. Struct., 11, 355 (2002).

14. S. D. Deshpande, J. Kim, and S. R. Yun, Synth. Met., 149, 53 (2005).

15. S. D. Deshpande, J. Kim, and S. R. Yun, Smart Mater. Struct., 14, 876 (2005).

16. S. Yonezawa, K. Nakamura, and Z. Takehara, J. Electrochem. Soc., 140, 629 (1993).

17. A. J. Motheo, E. C. Venancio, and L. H. Mattoso, J. Braz. Chem. Soc., 12, 526 (2001).

18. V. A. Bazhenov, "Piezoelectric Properties of Woods," Consultants Bureau, New York 1961.

19. D. Klemm, B. Heublein, H.-P. Fink, and A. Bohn, Angew. Chem., Int. Ed., 44, 3358 (2005). 